\title{
Communication
}

\section{Mutation analysis of large tumor suppressor genes LATS1 and LATS2 supports a tumor suppressor role in human cancer}

\author{
Tian Yu', John Bachman², Zhi-Chun Lai ${ }^{1,2,3 \bowtie}$ \\ ${ }^{1}$ Intercollege Graduate Degree Program in Molecular, Cellular and Integrative Biosciences, The Pennsylvania State \\ University, University Park, PA 16802, USA \\ 2 Department of Biology, The Pennsylvania State University, University Park, PA 16802, USA \\ ${ }^{3}$ Department of Biochemistry and Molecular Biology, The Pennsylvania State University, University Park, PA 16802, USA \\ $\triangle$ Correspondence: zcl1@psu.edu (Z.-C. Lai)
}

Received October 9, 2014 Accepted November 13, 2014

\begin{abstract}
In recent years, human cancer genome projects provide unprecedented opportunities for the discovery of cancer genes and signaling pathways that contribute to tumor development. While numerous gene mutations can be identified from each cancer genome, what these mutations mean for cancer is a challenging question to address, especially for those from less understood putative new cancer genes. As a powerful approach, in silico bioinformatics analysis could efficiently sort out mutations that are predicted to damage gene function. Such an analysis of human large tumor suppressor genes, LATS1 and LATS2, has been carried out and the results support a role of $h L A T S 1 / / 2$ as negative growth regulators and tumor suppressors.
\end{abstract}

KEYWORDS LATS1 \& LATS2, hippo signaling, cancer genome, human cancer

\section{INTRODUCTION}

Hippo signaling plays a crucial role in animal development and tumorigenesis (Harvey et al., 2013; Yu and Guan, 2013). As a key regulator of this growth-inhibitory pathway, the large tumor suppressor (Lats)/warts (wts) gene

Electronic supplementary material The online version of this article (doi:10.1007/s13238-014-0122-4) contains supplementary material, which is available to authorized users. encodes a Ser/Thr protein kinase and somatic mutations in human LATS1 and LATS2 have been identified in primary tumors (e.g. Murakami et al., 2011; Visser and Yang, 2010). To systematically evaluate how LATS1/2 genes play a critical role in human cancer, a mutation analysis has been carried out. In the Catalogue of Somatic Mutation in Cancer (COSMIC) database, 101 non-synonymous LATS1 somatic mutations have been identified from 9183 unique human tumor samples (Fig. 1A). Similarly, there are 80 LATS2 non-synonymous mutations out of 9516 samples (Fig. 1B). Therefore, an overall mutation rate is $1.10 \%$ for LATS1 and $0.84 \%$ for LATS2. In the cBioPortal database, LATS1 and LATS2 exhibited similar overall mutation rates, 1.83\% (135/ $7390)$ and $1.50 \%$ (111/7390), respectively. The top three highest mutation rates with relative larger total sample size for LATS 1 occurred in stomach adenocarcinoma $(5.91 \%$, $n=220)$, uterine corpus endometrial carcinoma $(4 \%, n=$ 248), and bladder urothelial carcinoma $(3.1 \%, n=130)$. Meanwhile, the highest LATS2 non-synonymous mutation rate occurred in uterine corpus endometrial carcinoma $(5.2 \%, n=248)$, stomach adenocarcinoma $(4.1 \%, n=220)$, and lung adenocarcinoma $(3.9 \%, n=229)$ (Table S1).

To determine the mutation distribution across different domains, analysis through Fisher's exact test shows that both the kinase domain $(P=0.01075)$ and proline-rich $(P=$ 0.0312 ) of LATS1 displayed the highest mutation frequency among all the LATS1 domains. The proline-rich domain had 7 mutations in a 31-amino acid (aa) region (2.2 mutations/10 aa), and the kinase domain had 43 mutations in a 306-aa region (1.41 mutations/10 aa) (Fig. 1A). In LATS2, the kinase domain $\left(P=5.66 \times 10^{-5}\right)$ and insertion domain $(P=0.03121)$ had the highest mutation frequency. LATS2 kinase domain 


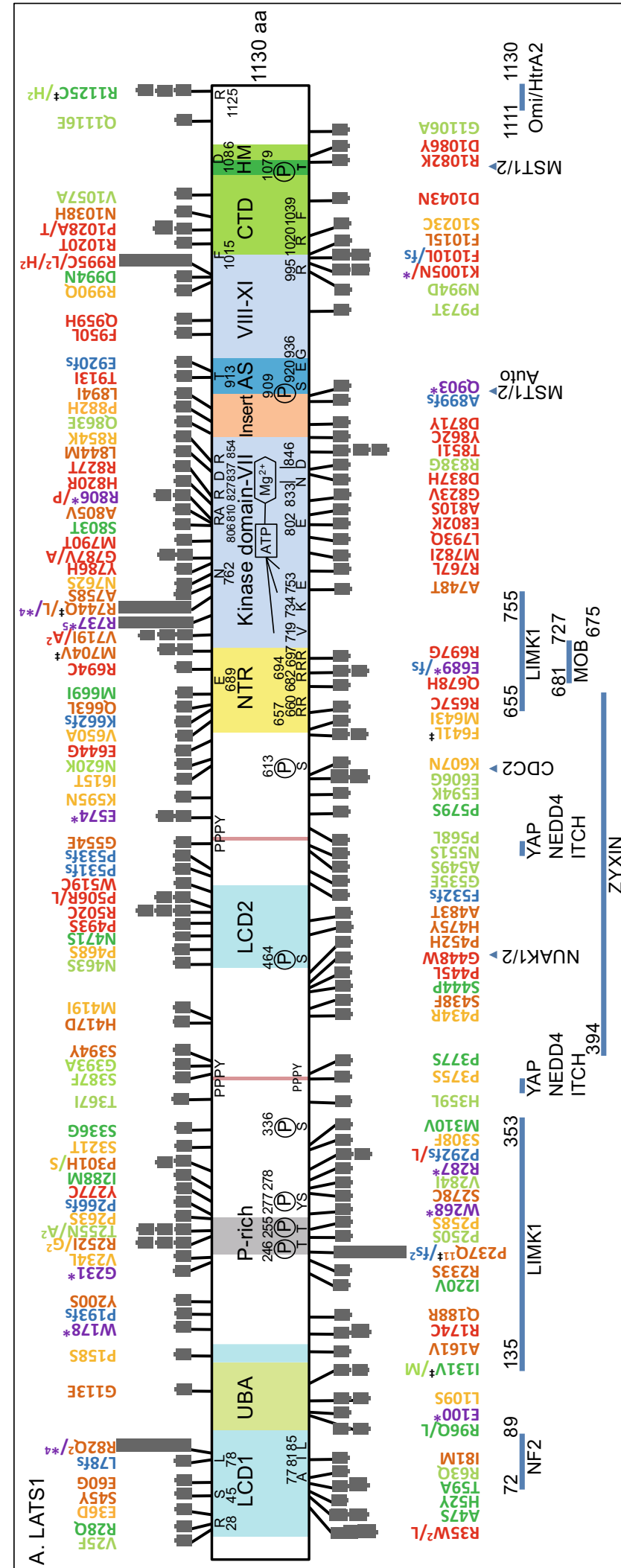

had 44 mutations in a 306-aa region (1.48 mutation/10 aa) and LATS2 insertion domain had 9 in 45 aa (2 mutation/10 aa) (Fig. 1B). These data support that selections have

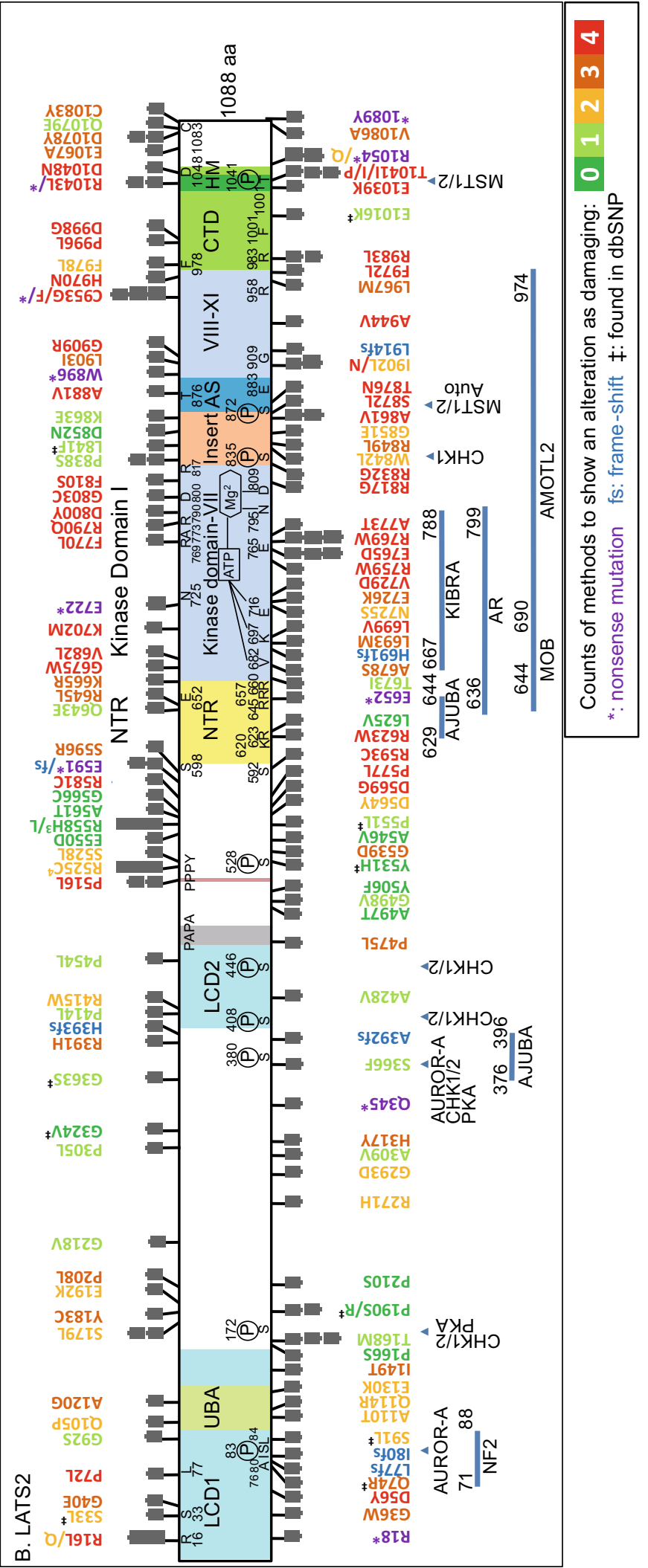

occurred to enrich mutations in functionally significant regions such as the kinase domain to facilitate tumorigenesis. 
Figure 1. Human cancer mutations in LATS1 (A) and LATS2 (B) are mapped to their corresponding open reading frames. Human LATS1 and LATS2 mutation data was collected from Catalogue of Somatic Mutations in Cancer (COSMIC) (top portions) and cBioPortal (bottom portions) databases. (A complete list of all mutations can be found in Table S1). All non-synonymous mutations are analyzed, using Uniprot identifier 095835 for LATS1 and Q9NRM7 for LATS2. Synonymous mutations were not included in this analysis. To evaluate potential impact of individual mutations on LATS $1 / 2$ structure and function, the following bioinformatics resources were used: 1) SIFT; 2) PROVEAN; 3) PolyPhen-2; and 4) Mutation Assessor. A color code was used to distinguish mutations that are predicted to be damaging by various numbers of the methods described above (zero in dark green, one in light green, two in orange yellow, three in brown, and four in red). "*” indicates nonsense mutation and " $¥$ " for ones found in dbSNP. "fs" is for frame-shift. Blue bars indicate regions involved in protein-protein interactions as indicated. Blue triangles identify phosphorylation sites by corresponding protein kinases. Each small block square indicates one unique mutation sample for LATS1/2 from human cancer.

Among all the mutations, nonsense and frame-shift mutations clearly disrupt LATS1/2 gene function. Thirteen and 10 unique nonsense mutations were found in LATS1 and LATS2, respectively. Moreover, 12 and 7 unique frameshift mutations were detected in LATS1 and LATS2, respectively (Fig. 1). The pattern of equal distribution of LATS1/2 nonsense or frame-shift mutations is consistent with the idea that LATS1 and LATS2 are tumor suppressor genes. The percentage of either nonsense or frame-shift mutations among all the mutations for LATS1 and LATS2, was $17.43 \%$ and $10.69 \%$, respectively.

To predict the functional relevance of other non-synonymous mutations in LATS1/2, we performed analyses of evolutionary conservation and protein structure through four different mutation-assessing methods: SIFT, PROVEAN, PolyPhen-2, and Mutation Assessor ( $\mathrm{Ng}$ and Henikoff, 2003; Adzhubei et al., 2010; Reva et al., 2011; Choi et al., 2012). We found that $73.85 \%(161 / 218)$ of missense mutations from LATS1 and $77.98 \%$ (124/159) from LATS2 are predicted to be damaging by at least one method (Fig. 1). In regards to mutations that were considered severe as determined by all four methods, LATS1 had a percentage of $25.69 \%(56 / 218)$ and LATS2 had 31.44\% (50/159).

\section{LCD1 AND LCD2}

LATS1/2 proteins share LATS conserved domain 1 (LCD1) and LATS conserved domain 2 (LCD2), which are conserved in all vertebrate LATS1/2 homologues. LCD1 and LCD2 are critical for LATS1/2 function and regulation. The deletions of either LCD1 or LCD2 in mouse Lats2 abolished its tumor suppressor activity in immortalized mouse cell line (Visser and Yang, 2010). Lats1 LCD1 knockout mice were born with a low birth rate, from which the mouse embryonic fibroblasts displayed chromosomal instability and tumorigenesis (Yabuta et al., 2011). Within LCD1, a short segment called Conserved N-terminal Motif (CNM) (aa 72-89 for LATS1 and 71-88 for LATS2) is important for membrane recruitment and activation of LATS $1 / 2$ by Merlin/NF2. The alterations of three highly conserved residues in LATS1/2-A77/76P-I81/80T-L85/84P prevented its interaction with Merlin/NF2, membrane localization and activation (Yin et al., 2013). Therefore, LATS1-I81M, LATS1-R82Q, and LATS2-P72L mutations may fail to interact with Merlin/NF2 and consequently cannot be activated (Fig. 1). The truncated products of LATS1 such as L78fs, R82*, E100*, W178*, and G231* may compete with wild-type LATS proteins for the binding partners of LCD1. Interestingly, LATS2-S83 within CNM is phosphorylated by Aurora-A to regulate the centrosomal localization and mitotic activity of LATS2 (Visser and Yang, 2010). Finally, the conserved residues in LCD1 could be critical for function. Mutations were found at certain conserved sites in LCD1 which include LATS1/2-R28/R16, S45/S33, L78/L77, and 181/l80. Moreover, N463S, P468S, H475Y, A483T, P493S, R502C, P506R/L, and W519C in LCD2 for LATS1 are predicted to be damaging. P468S is close to the phosphorylation site LATS1-S464 by NUAK, which promotes LATS1 degradation. Additionally, CDC2 phosphorylates $\mathrm{S} 613$ of LATS1. CDC2 forms a complex with LATS1 in the centrosome and phosphorylation of S613 occurs during mitosis (Visser and Yang, 2010). Mutations near S613, such as K607N and I615T, may interfere with phosphorylation at this site. Four lesser deleterious LATS2 mutations were found in LCD2, which contains the phosphorylation sites $\$ 408$ and $\$ 446$ by Chk1/2 in response to UV damage (Okada et al., 2011). Next to LCD2, LATS2-S380 is phosphorylated by Aurora A during mitosis, which is critical for Aurora A-LATS-Aurora $B$ axis to regulate mitotic progression (Yabuta et al., 2011). Moreover, LATS2 S380 is located within an Ajuba-binding region of LATS2 (aa 376-396), which regulates the spindle apparatus formation (Visser and Yang, 2010). Mutations like $\mathrm{R} 391 \mathrm{H}$ may affect the interactions and cell cycle control.

\section{PROLINE-RICH REGION}

LATS1 has a unique proline-rich region (Fig. 1A). Previous studies detected phosphorylation of T246 and T255 in P-stretch, as well as S336 located downstream (Hornbeck et al., 2012). T255A/N and S336G mutations in LATS1 would prevent phosphorylation of these residues, and the R252I/G mutations nearby may affect these phosphorylation events. Moreover, Y277 and S278 located within the LIMK-binding site of LATS1 were also found to be phosphorylated (Visser and Yang, 2010). While the functional significance of these phosphorylations is unknown, Y277C 
and S278C mutations clearly prevent phosphorylation of these two residues.

\section{BINDING WITH YAP AND KIBRA}

The interaction between the Proline-Proline-x-Tyrosine (PPxY) motif and the hydrophobic pocket of WW domain is critical for the LATS1/2 binding with either their substrate YAP or their activator KIBRA. It has been reported that LATS1-Y559F and Y376A abrogated the binding with YAP (Visser and Yang, 2010), while LATS2-Y518A partially abolish the binding with KIBRA (Xiao et al., 2011). These interactions may be affected by the third proline mutation P375S in the first PPXY motif of LATS1 and the second proline mutation P516L in the only PPxY motif of LATS2.

\section{BINDING WITH MOB, AJUBA AND AMOTL2}

The N-terminal regulatory domain (NTR) adjacent to the kinase domain is required for LATS1/2 activation by MOB, AJUBA, and AMOTL2 (Visser and Yang, 2010; Paramasivam et al., 2011; Xiao et al., 2011). Positively charged residues such as LATS1-R657/LATS2-K620, R660/R623, R682/ R645, R694/R657, and R697/R660 in LATS1/2 NTR are conserved in the NDR subfamily from yeast to human. Previous studies have shown that LATS1 mutations (e.g. R657A, R660A and R694A) abolish their interaction with the negatively charged surface of MOB1A/B and kinase activity (Hergovich et al., 2006). Therefore, LATS1 mutants (R657C, R694C and R697G) and LATS2 mutants (R623W and $\mathrm{R} 645 \mathrm{~L})$, are expected to become inactive in human cancer due to loss of interactions with LATS activators such as MOB. The Drosophila Wts-R702 residue is equivalent to LATS1/2-R694/R657 and critical for Mob-binding, kinase activity, and inhibition of tissue growth in development (Ho et al., 2010).

\section{THE KINASE DOMAIN}

LATS1 and LATS2 are members of the AGC (named after PKA, PKG, and PKC) protein kinase family. Although crystal structures of some AGC proteins have been deciphered, there is no structure information available for LATS1/2 kinase domains. To estimate mutation-induced structural changes, we performed structure remodeling for LATS1/2 based on two AGC family proteins, ROCK and PKC. Conserved in most eukaryotic protein kinases, the $\mathrm{N}$-terminal catalytic domain of LATS1/2 interacts with the phosphate donor ATP through a crucial network, composited by GxGxxGxV loop (LATS1: 712-719/ LATS2: 675-682), K734/697, E753/716, DxKxxN (828-833/791-795) and DFG motif (846-848/809811) ("x" represents any aa) (Endicott et al., 2012; Hanks and Hunter, 1995). In addition, previous studies also have verified that mutants LATS1-K734M/LATS2-K697A/mLats2K655M are all kinase-dead (Zhao et al., 2007; Wei et al., 2007; Visser and Yang, 2010). Mutated within or close to these conserved catalytic elements, LATS1 V719I/A, $\mathrm{R} 744 \mathrm{Q} / \mathrm{L}, \mathrm{A} 748 \mathrm{~T}, \mathrm{R} 827 \mathrm{~T}, \mathrm{R} 837 \mathrm{H}, \mathrm{L} 844 \mathrm{M}$ and LATS2 G675W, A678S, V682L, L693M, L699V, D800Y, G803C are most likely to disrupt ATP binding and catalysis. LATS1R995C/L/H and LATS2-G909R also change highly conserved residues. Therefore, these cancer mutants may affect their kinase activity. Moreover, conserved residues in the kinase domain of LATS1/2 are expected to be important for their kinase activity. Mutations occurring in these residues included LATS1-N762/LATS2-N725, R806/R769, A810/ A773, R827/R790, D837/D800, R854/R817, and R995/R958 (Fig. 1).

Unlike other AGC family members, LATS1/2 and the NDR subfamily members have an insert between the kinase subdomain VII and the activation segment (AS) in the subdomain VIII. Basic residues in the insert inhibit the activity of AS likely through an auto-inhibitory mechanism (Hergovich et al., 2006). Missense mutations in this region (e.g. LATS1-D871Y) may have an impact on the kinase activity (Fig. 1).

\section{PHOSPHORYLATION AND ACTIVATION BY MST1/2 KINASES}

The phospho-S909/S872 and other residues in AS organize interaction between ATP and substrates of LATS1/2. Previous study has shown that LATS1 mutant S909A/D/E cannot be activated by the MST2 kinase. Therefore, LATS2-S872L would abolish the kinase activity, and LATS1-T913I and LATS2-T876N, A881V also likely damage catalytic activity. In the C-terminal of kinase IX and XI subdomains, the LATS2G909 and LATS1-R995 are highly conserved among eukaryotic kinases (Endicott et al., 2012). LATS1-R995C/L/H and LATS2-G909R would probably damage kinase activity. LATS2-G909R and C953* has been experimentally shown to be defective for kinase activity and YAP regulation (Yu et al., 2013).

In the C-terminal domain (CTD) of AGC family kinase, the conserved NFD (Asn-Phe-Asp) motif interacts with hydrophobic pocket in the $\mathrm{N}$-terminus of kinase domain to facilitate kinase activation (e.g. PKC, Leonard et al., 2011). In LATS1 NFD, N1038H may disrupt the kinase activity. In addition, LATS1-R1020T, P1028A/T, S1023C and LATS2P996L, D998G may also affect this activation. LATS1/2 activation also requires phosphorylation of T1079/T1041 by MST1/2 in hydrophobic motif (HM), which has a consensus sequence $F-x-x-Y / F-T-Y / F-K / R$ in the NDR protein subfamily (Hergovich et al., 2006). Thus, LATS2-T1041I/P mutations clearly abolish phosphorylation at this site to cause kinase inactivation. LATS1-R1082K, D1086Y and LATS2-E1039K, R1043L, D1048N may disrupt kinase activity as well. Mutations at conserved sites between LATS1/2 such as LATS1-F1015/LATS2-F978, R1020/R983, F1039/F1001, and D1086/D1048 may affect function of the C-terminus. 


\section{CONCLUDING REMARKS}

Genetic analysis of Lats/Wts family genes using Drosophila and mice models has revealed their role as negative growth regulators and tumor suppressors in animal (Visser and Yang, 2010; Harvey et al., 2013; Yu and Guan, 2013). The fact that human LATS1 can functionally replace Wts in Drosophila supports that LATS may function as a tumor suppressor in human cells. From human cancer genome projects, an increasing number of mutations in LATS1/2 are detected. Compared to some well-established cancer genes such as TP53 and Rb, LATS1 and LATS2 are not frequently mutated. However, our in silico analysis provides supporting evidence that LATS1/2 mutations drive human tumor development based on the following observations: 1) Cancer mutations in $h L A T S 1 / 2$ do not appear to be random mutations. Damaging mutations have been accumulated more preferentially in important protein domains such as the kinase domain. Majority of the mutations including nonsense and frame-shift mutations clearly disrupt LATS1/2 function; 2) Some mutations occurred in regions and residues important for LATS1/2 activation. Examples include MST1/2 phosphorylation sites, MOB-binding domain, and the region critical for NF2 interaction; 3) In certain cancer types like stomach adenocarcinoma and uterine corpus endometrial carcinoma, mutation rates can be reasonably high $(5.2 \%-$ $5.9 \%$ ). On the other hand, mechanisms other than gene mutation could also be effective to alter gene activity. In this regard, LATS1/2 genes are known to be down-regulated by promoter methylation. Mutations in other Hippo pathway genes such as MST1/2 are also expected to reduce LATS1/2 function. While the functional significance of some of these mutations have been experimentally tested in vivo (Yu et al., 2013), results reported here further support that $L A T S 1 / 2$ act normally as tumor suppressors and loss of their functions contributes to human cancer development.

\section{ACKNOWLEDGEMENTS}

This work was partly supported by the National Science Foundation. We would like to thank Nicholas Anzalone and Samar Almarzooqi for assistance and other members of the Lai laboratory for discussions.

\section{ABBREVIATIONS}

CTD, C-terminal domain; HM, hydrophobic motif; LCD1, LATS conserved domain 1; LCD2, LATS conserved domain 2; NTR, $\mathrm{N}$-terminal regulatory domain; PPxY, proline-proline-x-tyrosine.

\section{COMPLIANCE WITH ETHICS GUIDELINES}

Tian Yu, John Bachman, and Zhi-Chun Lai declare that they have no conflict of interest. This article does not contain any studies with human or animal subjects performed by the any of the authors.

\section{OPEN ACCESS}

This article is distributed under the terms of the Creative Commons Attribution License which permits any use, distribution, and reproduction in any medium, provided the original author(s) and the source are credited.

\section{REFERENCES}

Adzhubei IA, Schmidt S, Peshkin L, Ramensky VE, Gerasimova A, Bork P, Kondrashov AS, Sunyaev SR (2010) A method and server for predicting damaging missense mutations. Nat Methods 7:248-249

Choi Y, Sims GE, Murphy S, Miller JR, Chan AP (2012) Predicting the functional effect of amino acid substitutions and indels. PLoS One 7:e46688

Endicott JA, Noble ME, Johnson LN (2012) The structural basis for control of eukaryotic protein kinases. Annu Rev Biochem 81:587613

Hanks SK, Hunter T (1995) Protein kinases 6. The eukaryotic protein kinase superfamily: kinase (catalytic) domain structure and classification. FASEB J 9:576-596

Harvey KF, Zhang X, Thomas DM (2013) The Hippo pathway and human cancer. Nat Rev Cancer 13:246-257

Hergovich A, Stegert MR, Schmitz D, Hemmings BA (2006) NDR kinases regulate essential cell processes from yeast to humans. Nat Rev Mol Cell Biol 7:253-264

Ho LL, Wei X, Shimizu T, Lai ZC (2010) Mob as tumor suppressor is activated at the cell membrane to control tissue growth and organ size in Drosophila. Dev Biol 337:274-283

Hornbeck PV, Kornhauser JM, Tkachev S, Zhang B, Skrzypek E, Murray B, Latham V, Sullivan M (2012) PhosphoSitePlus: a comprehensive resource for investigating the structure and function of experimentally determined post-translational modifications in man and mouse. Nucleic Acids Res 40:D261-D270

Leonard TA, Różycki B, Saidi LF, Hummer G, Hurley JH (2011) Crystal structure and allosteric activation of protein kinase $C \beta I I$. Cell 144:55-66

Murakami H, Mizuno T, Taniguchi T, Fujii M, Ishiguro F, Fukui T, Akatsuka S, Horio Y, Hida T, Kondo Y, Toyokuni S, Osada H, Sekido $Y$ (2011) LATS2 is a tumor suppressor gene of malignant mesothelioma. Cancer Res 71:873-883

$\mathrm{Ng}$ PC, Henikoff S (2003) SIFT: predicting amino acid changes that affect protein function. Nucleic Acids Res 31:3812-3814

Okada N, Yabuta N, Suzuki H, Aylon Y, Oren M, Nojima H (2011) A novel Chk1/2-Lats2-14-3-3 signaling pathway regulates P-body formation in response to UV damage. J Cell Sci 124:57-67

Paramasivam M, Sarkeshik A, Yates JR, Fernandes MJ, McCollum D (2011) Angiomotin family proteins are novel activators of the LATS2 kinase tumor suppressor. Mol Biol Cell 22:3725-3733

Reva B, Antipin Y, Sander C (2011) Predicting the functional impact of protein mutations: application to cancer genomics. Nucleic Acids Res 39:e118

Visser S, Yang X (2010) LATS tumor suppressor: a new governor of cellular homeostasis. Cell Cycle 9:3892-3903

Wei X, Shimizu T, Lai ZC (2007) Mob as tumor suppressor is activated by Hippo kinase for growth inhibition in Drosophila. EMBO J 26:1772-1781

Xiao L, Chen Y, Ji M, Dong J (2011) KIBRA regulates Hippo signaling activity via interactions with large tumor suppressor kinases. J Biol Chem 286:7788-7796 
Yabuta N, Mukai S, Okada N, Aylon Y, Nojima H (2011) The tumor suppressor Lats2 is pivotal in Aurora A and Aurora B signaling during mitosis. Cell Cycle 10:2724-2736

Yin F, Yu J, Zheng Y, Chen Q, Zhang N, Pan D (2013) Spatial organization of Hippo signaling at the plasma membrane mediated by the tumor suppressor Merlin/NF2. Cell 154:1342-1355

Yu FX, Guan KL (2013) The Hippo pathway: regulators and regulations. Genes Dev 27:355-371
Yu T, Bachman J, Lai ZC (2013) Evidence for a tumor suppressor role for the large tumor suppressor genes LATS1 and LATS2 in human cancer. Genetics 195:1193-1196

Zhao B, Wei X, Li W, Udan RS, Yang Q, Kim J, Xie J, Ikenoue T, Yu J, Li L, Zheng P, Ye K, Chinnaiyan A, Halder G, Lai ZC, Guan KL (2007) Inactivation of YAP oncoprotein by the Hippo pathway is involved in cell contact inhibition and tissue growth control. Genes Dev 21:2747-2761 